ENCYCLOPÉDIE Encyclopédie berbère

BERBERE $\quad \mathbf{1 4} \mid \mathbf{1 9 9 4}$

14 | Conseil - Danse

\title{
Cuirs et peaux
}

M. Gast

\section{OpenEdition}

Journals

Édition électronique

URL : http://journals.openedition.org/encyclopedieberbere/2346

DOI : 10.4000/encyclopedieberbere.2346

ISSN : 2262-7197

\section{Éditeur}

Peeters Publishers

\section{Édition imprimée}

Date de publication : 1 septembre 1994

Pagination : 2144-2153

ISBN : 2-85744-741-8

ISSN : 1015-7344

\section{Référence électronique}

M. Gast, « Cuirs et peaux », Encyclopédie berbère [En ligne], 14 | 1994, document C108, mis en ligne le

01 mars 2012, consulté le 10 décembre 2020. URL : http://journals.openedition.org/

encyclopedieberbere/2346; DOI : https://doi.org/10.4000/encyclopedieberbere.2346

Ce document a été généré automatiquement le 10 décembre 2020.

(c) Tous droits réservés 


\section{Cuirs et peaux}

\section{Gast}

1 Le cuir se nomme êyit en tamahaq, aglim en mozabite et en ouargli, agwlim et afilali en kabyle. La peau porte soit les mêmes noms, soit une gamme très variée de dénominations en rapport avec ses usages.

2 La peau et le cuir ont servi depuis des temps immémoriaux les besoins de l'homme; mais il est difficile de définir l'âge de leurs premiers usages étant donné leur matière essentiellement périssable. Seule l'archéologie peut nous donner des témoignages précis dans les sépultures et sur les peintures et gravures rupestres. Les Egyptiens enveloppaient le corps mortel des hommes dans des peaux (auxquelles fut substituée plus tard le linceul) pour lui assurer le passage vers la lumière, c'est-à-dire vers l'immortalité. Dans le Tibesti, M. Dalloni découvre en 1930-31 des cadavres en position contractée, enveloppés dans des peaux de bœufs maintenues avec des épines d'acacia. Dans l'Ahaggar des observations semblables ont été faites dans la Tefedest, à Abalessa dans le tombeau de Tin Hinan* (470 après J.-C), dans l'Adrar Ahnet, et au Niger dans la région d'In Gall (Fouilles, Paris). Les peintures et gravures rupestres de l'Afrique du Nord et du Sahara abondent en figurations de personnages masculins et féminins portant des vêtements (pagnes, ceintures, jupes, baudriers, chaussures) des boucliers ou autres attributs qui paraissent selon toute vraisemblance en cuir. 


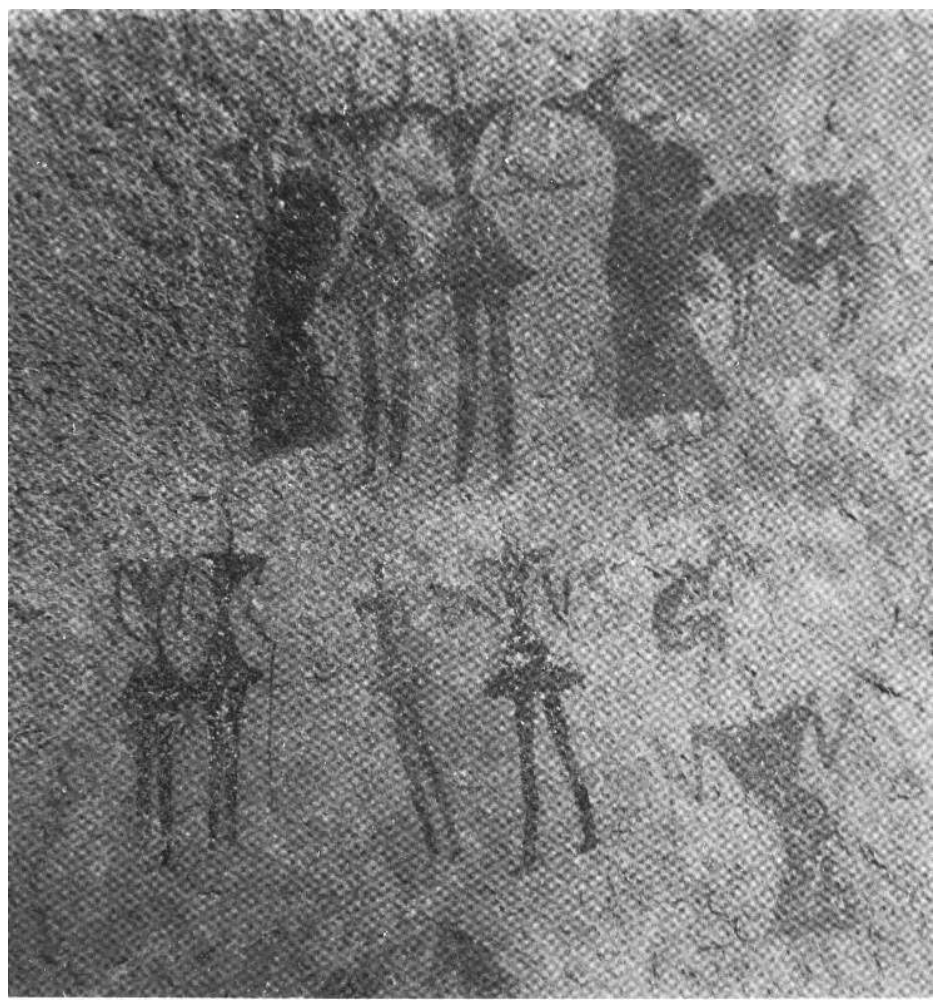

Les personnages portent vraisemblablement des vêtements de cuir (Photo M. Gast)

3 Il arrive même que les détails de vêtements portant des décorations de franges de cuir ou de chaussures, soient d'une finesse et d'une précision ethnographique étonnantes, comme dans la grotte de Tahilahi au Tassili-n-Ajjer. Il est évident qu'avant l'apparition des tissages, les hommes de la préhistoire usaient abondamment de peaux d'animaux sauvages ou domestiques pour satisfaire de nombreux besoins. Jusqu'en 1940 les montagnards les plus pauvres de l'Ahaggar se vêtissaient encore de la tébétiq, tunique de peau à franges qui donne une silhouette semblable à celle des personnages appelés «bitriangulaires" ou " en diabolo » sur les peintures rupestres du Sahara. Mais en période de famine ces mêmes populations sahariennes débitent les peaux fraîches en fines lanières, les font bouillir après les avoir dépouillées de leurs poils, et s'en nourrissent ainsi que des vieux morceaux de cuir ou de peaux cuits sous la cendre et qu'on nomme abañyôr (ibeñyân). Les jeunes nomades du Sahara Central portent encore parfois un caleçon de cuir appelé argag et les bergers Peuls de la boucle du Niger jusqu'au Tamesna, sont toujours vêtus d'une solide jupe de cuir qu'ils préfèrent aux vêtements de coton indigo plus fragiles dans les travaux d'exhaure de l'eau qu'ils pratiquent presque journellement.

4 Cet art du vêtement de cuir des «Libyens » aurait été emprunté par les Grecs selon Hérodote. L'égide d'Athena serait un manteau de peaux de chèvres (avec leur fourrure) comme en portaient récemment les nomades du Sahara Central et qui servait aussi de tapis pour dormir. L'on peut émettre l'hypothèse d'une affinité d'usage et de formes entre ce manteau de peaux et le burnous actuel dont la découpe particulière semble tout à fait aberrante sur un métier à tisser. La forme et l'usage du burnous qui campent bien les Berbères d'Afrique du Nord, paraissent avoir été conçus en peaux avant de 
devenir ce vêtement de laine tissée, entièrement fabriqué dans les familles et, jusqu’à ce jour, jamais industrialisé.

Berger vêtu d'un caleçon de cuir abreuvant ses bœufs à l'aide d'un délou (puisette en cuir), à In Abangarit (Photo M. Gast)

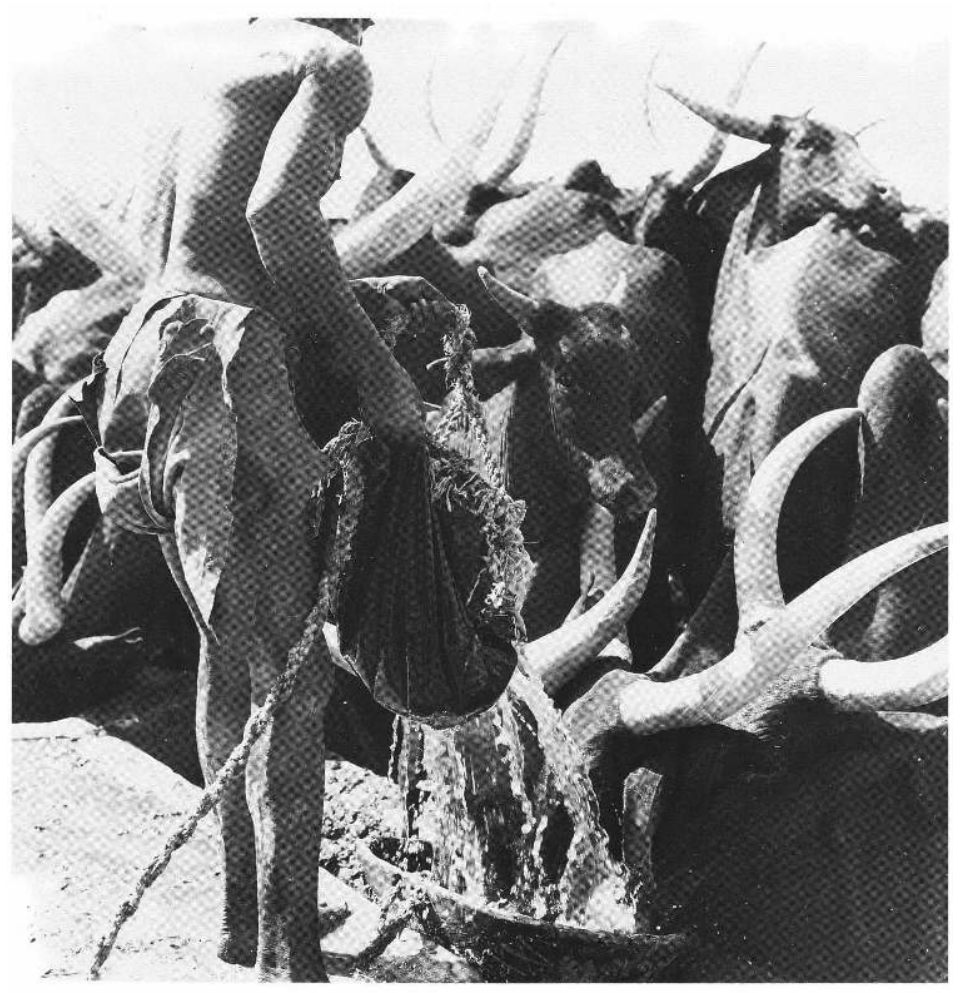

$5 \quad$ Alors qu'en milieu urbain le travail des peaux s'est inséré dans les cités, en corporations bien structurées comme dans le Tafilalet au Moyen Age, à Tunis, à Marrakech et Fès jusqu'à notre époque, en milieu essentiellement berbère, tant chez les nomades que chez les sédentaires, le travail des peaux reste une activité artisanale parmi d'autres. Il est soit l'apanage d'artisans à la fois forgerons, bijoutiers, sculpteurs, voire potiers qui fabriquent des objets utilitaires en métal, en bois (en écorce), en poterie, en cuir, en peau ou en os, et aussi des bijoux, soit ressortissant d'activités féminines en milieu rural ou chez les nomades. Alors que dans ce dernier cas le travail du cuir et des peaux est essentiellement réservé aux femmes, dès qu'il devient un métier rémunérateur, organisé en corporation, il reste exclusivement réservé aux hommes. Dans le cas des corporations qui traitent des quantités industrielles de peaux, la société des artisans est non seulement hiérarchisée selon le niveau de spécialisation des ouvriers et de leur savoir-faire, mais elle est aussi structurée autour d'un code de valeurs morales, une pensée cosmogonique, une organisation spatiale et technique des différentes opérations traitant les peaux. Les artisans sont soumis au secret professionnel, car la survie de la corporation en dépend (voir D. Jemma, Les tanneurs de Marrakech, 1971). Cette organisation permet une production abondante et variée qui s'exporte très loin au-delà des frontières du pays. Ce fut le cas des peaux du Tafilalet dont la qualité explique le nom de filali donné aux peaux finement tannées. Le Maroc étant depuis longtemps le pays du tannage des peaux, le maroquin désigne la peausserie de chèvre de tannage végétal et la maroquinerie, la fabrication de petits objets en cuir ou ces objets eux-mêmes. 


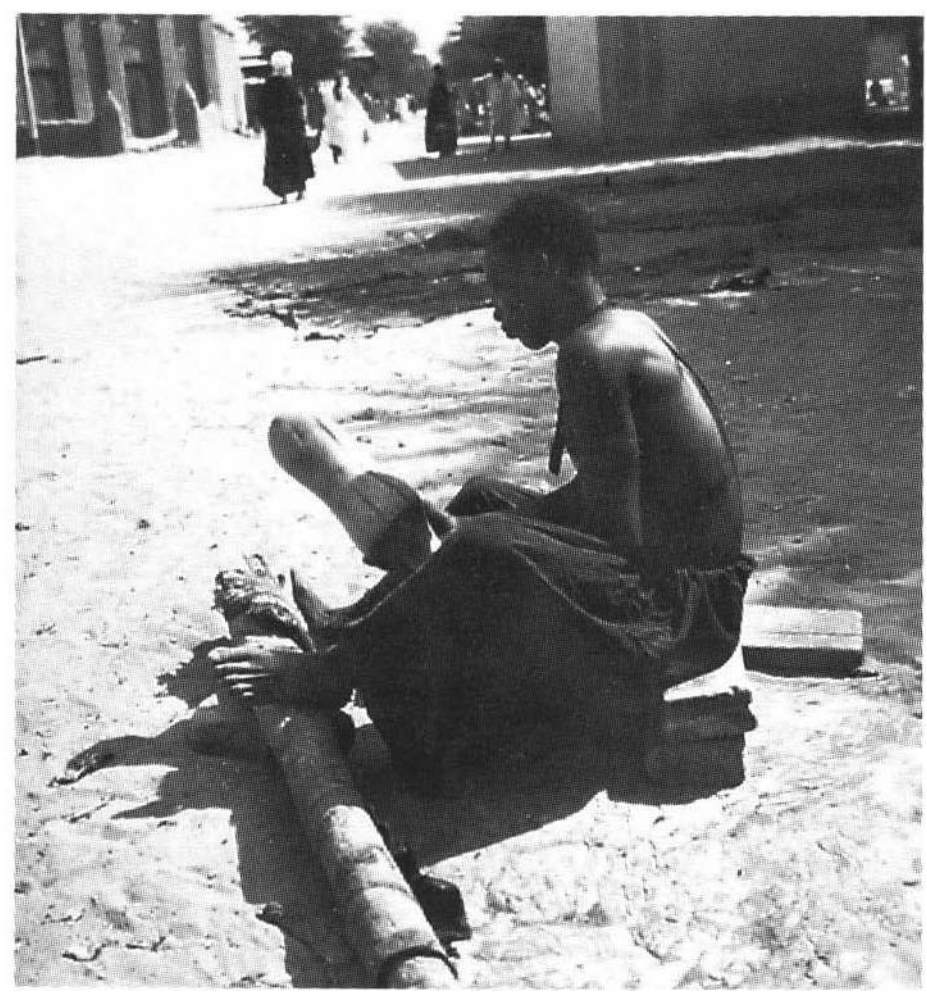

6 L'artisanat nomade ou familial en milieu rural ne se situe pas au même niveau; il reste un savoir collectif, accessible à tout le monde, pour satisfaire les besoins domestiques internes. Cependant, dans les sociétés sahariennes, il existe encore dans certaines régions, des artisans savetiers spécialisés comme dans le Touat, le Tidikelt et le Gourara (qui font des chaussures de marche appelées belya), d'autres artisans du cuir fabriquent des sandales et autres objets en cuir à Agadez, In Gall, Tahoua, Gao dans les régions subsahariennes, avec une spécialité particulière à Agadez: la selle touarègue pour dromadaire, faite d'un assemblage de planchettes et de cuir avec un pommeau en forme de croix (voir Th. Monod 1967).

7 Chez les Touaregs, les artisans ou Enaḍen forment un clan en principe endogame, spécialisé dans l'art du feu, du bois et du cuir. Là, ce sont les femmes qui tannent, confectionnent et décorent les objets en cuir (sacs de peau, rênes de chameaux, cordons de pantalon, lacets de suspension, porte-feuilles, porte-amulettes, etc.). Mais ces travaux de cuir ne leur sont pas exclusifs. Toutes les femmes nomades quel que soit leur rang, les pratiquent plus ou moins, et c'est une tradition d'offrir leurs ouvrages aux invités, aux personnes qu'elles estiment et qu'elles veulent honorer. 


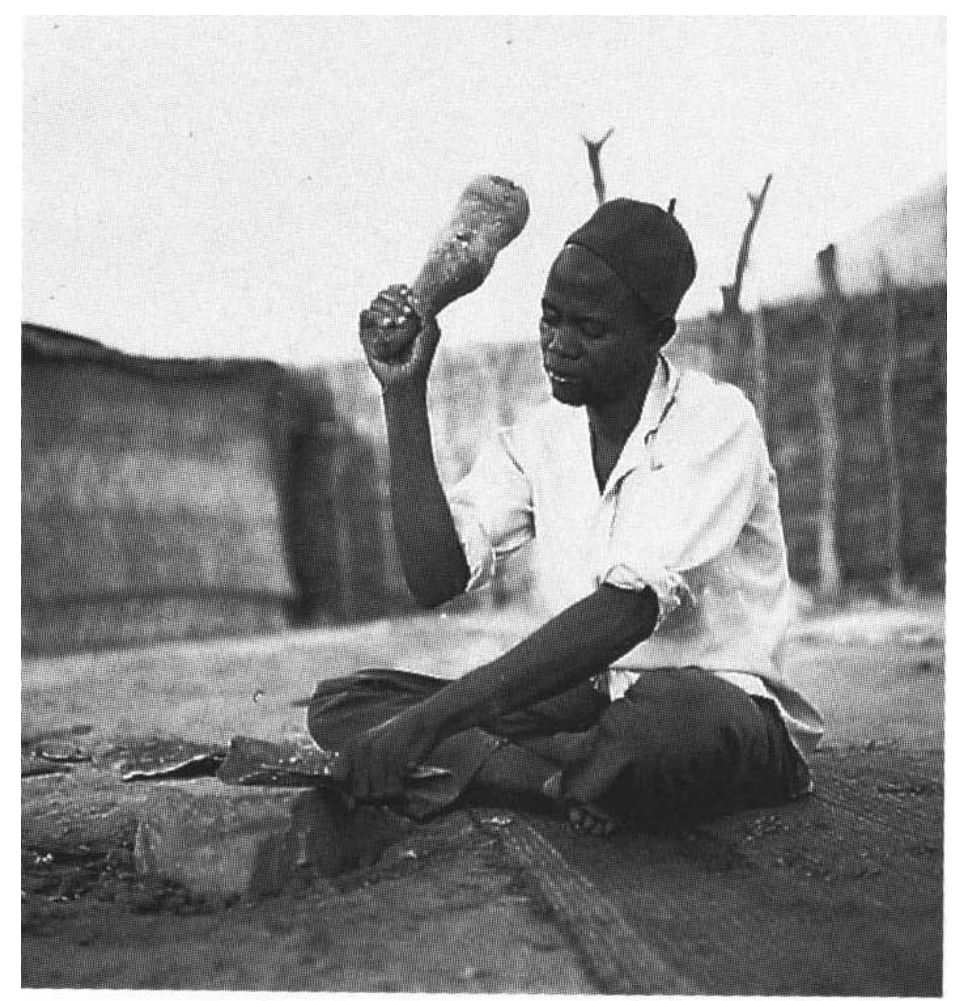

\section{Les peaux crues (sans aucune préparation)}

8 La peau crue avec ou sans poil est utilisée à différents usages qui varient selon l'animal qui l'a fournie.

9 La peau de girafe, la peau d'oryx étaient utilisées comme boucliers en raison de leur épaisseur et de leur résistance à la perforation. La peau de chameau qui ne donne pas de bon cuir, sert à confectionner des outres à beurre, des bacs en peau rigide pour le tannage ou comme abreuvoir, ou, découpée en lanières pour confectionner des entraves d'animaux et des liens pour serrer des pièces de bois (lits de nervures de palme, supports plats, bâts de chameau ou d'âne).

10 La peau de bœuf plus appréciée que la précédente comme lien, peut participer en outre à la fabrication de chaussures assujetties au pied à l'aide de lacets crus ou tannés. Ce type de chaussures rustiques, bien que de conception différente, est connu des régions telliennes du nord jusqu'en Afrique au sud du Sahara, comme dans de nombreuses régions du monde. En climat saharien où l'air est très sec, ces peaux crues acquièrent la rigidité du bois et il est difficile de les dénouer ou les découper quand elles sont sèches.

11 La peau crue de bœuf en climat sec a pu suppléer à l'absence de pièces métalliques dans la fabrication d'engins techniques comme dans les roues en bois et des appareillages permettant l'exhaure de l'eau par traction animale (voir délou*), mais aussi à l'époque proto-historique dans la fabrication des chars attelés de chevaux chez les Garamantes et autres populations du Sahara Central et du Maghreb (Jean Spruytte, 1977). La peau crue de bœuf a joué à travers les siècles le rôle technique qu'on accorde aujourd'hui à la tôle de fer et au fil de fer comme matériaux à usages multiples. Cependant, elle conserve encore aujourd'hui un avantage sur le métal quand il s'agit d'assembler des 
pièces de bois. Tout en maintenant une parfaite rigidité aux éléments qu'elle assujettit, la peau crue, en climat sec, garde la souplesse minimale qui évite aux pièces ainsi assemblées de se briser sous des efforts violents. Ce mode d'assemblage a aussi l'avantage de pouvoir être réparé, en cas de besoin, facilement avec les moyens du bord.

La peau de chèvre peut aussi être utilisée crue comme lacet ou attache, mais étant donné ses grandes qualités plastiques et sa solidité, elle est plutôt réservée pour être tannée. Cependant elle est très appréciée comme membrane d'instruments à percussion (tam-tam de toutes sortes, darbouka, tambours) et en pays touareg sur le grand mortier de bois (tindé).

Peau de mouflon remplie de sable humide, après tannage, pour lui rendre sa forme. Elle est destinée au transport des denrées sèches, Ahaggar (Photo M. Gast)

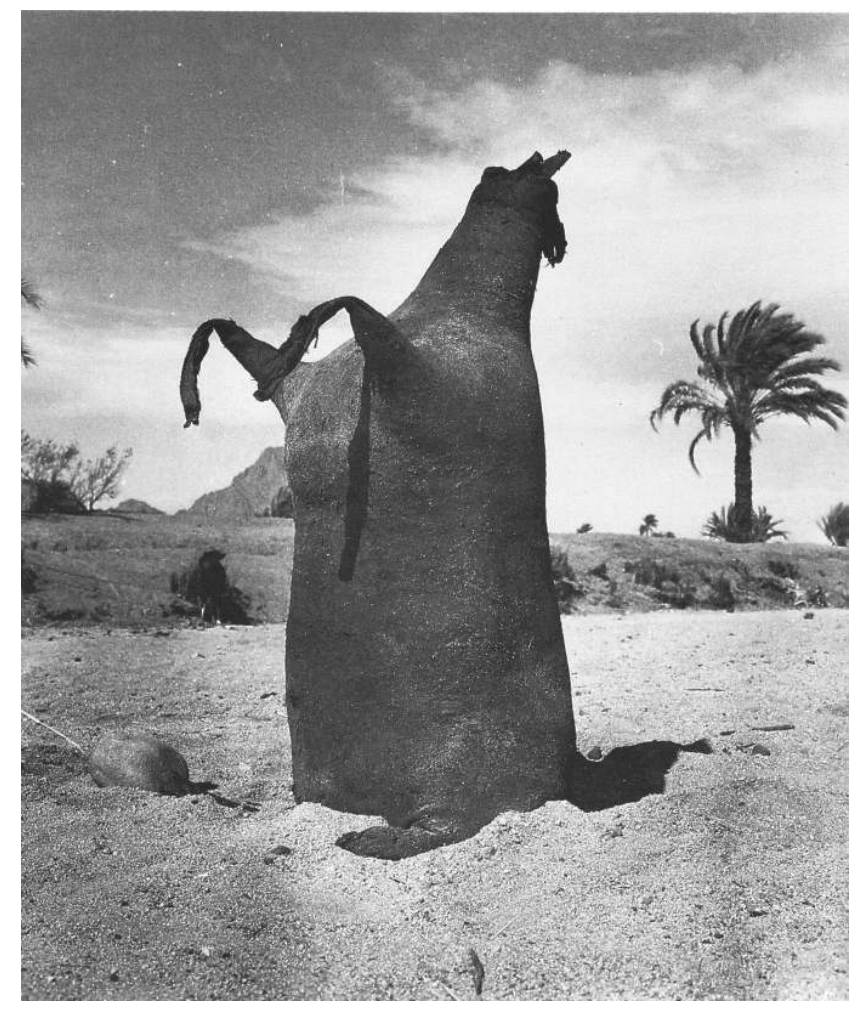

13 La peau crue de mouton n'a guère d'usage car elle se révèle fragile, cassante, peu résistante ; elle ne possède pas la texture de fibres qui fait la qualité des peaux de bœuf ou de chèvre mais tannée, elle acquiert des qualités qui la rendent apte à de multiples usages.

\section{Les peaux tannées}

Chaque région a ses recettes de tannage qui varient selon la disponibilité des produits tannants, mais aussi selon des savoirs locaux anciens en rapport avec l'écologie locale (végétaux, minéraux d'extraction régionale).

Il s'agit d'abord de savoir si l'on désire garder une peau avec sa toison ou si elle doit être épilée; si elle doit servir d'enveloppe sèche ou de contenant de produits liquides. Les manteaux de peau, les tapis ou couvertures (faro), les petites peaux de mouton qui 
participent à la confection de gilets de laine, de tapis pour dormir (ağlim), subissent un tannage spécial qui sauvegarde la toison. Les vêtements à usage domestique sont toujours traités et conçus en petites quantités par les femmes sous la tente ou dans les maisons. Les surplus de production sont vendus par les hommes.

L'outre à eau dont on conserve les poils à l'extérieur, est préparée différemment au Mzab (où elle est enduite de goudron végétal issu du genévrier), au Sahara Central (enduite de beurre) ou en Kabylie.

Etre tanné se dit afel en tamâhaq; tafelt désigne le tan. Celui-ci peut être constitué à partir d'écorces d'arbres (Acacia Seyal, Acacia Raddiana, Rhus oxyacantha), de galles (galle de Tamaris aphylla) de fruits (Acacia arabica), de feuilles (Leptadenia pyrotechnica). Mais l'alun et le natron ainsi que le chlorure de sodium (gros sel) sont aussi très employés selon les régions. Après avoir été minutieusement dégraissée, grattée à l'aide d'une pierre, lavée et malaxée, la peau munie de sa fourrure peut être desséchée avec une couche de sel, de natron ou d'alun ou simplement recevoir un corps gras pour l'assouplir (pour les petites peaux d'animaux).

Grand sac en peau crue de chameau (ahağa), outre pour baratter le lait et outre à eau (ağivir) dans un campement touareg (Photo M. Gast)

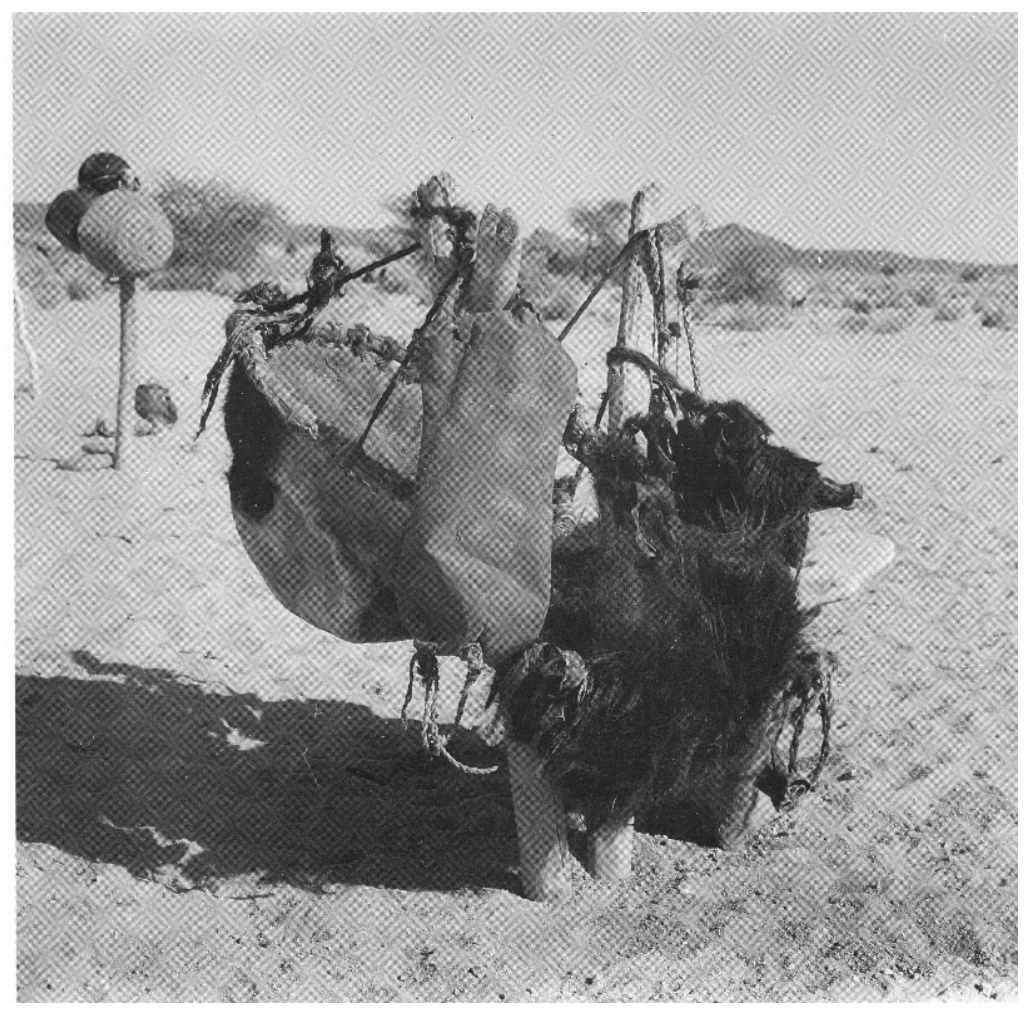

Toutes les autres peaux qui doivent être épilées sont immergées un ou plusieurs jours dans une solution épilatoire dont le choix varie selon la destination de la peau. Dans le Sahara Central les nomades emploient Pergularia Tomentosa (tachkat en tamâhaq) et Leptadenia pyrotechnica (ana); mais s'il s'agit de préparer les petites outres à contenir ou faire cailler le lait, les femmes touarègues préféraient utiliser la cendre de bois mélangée à du sel pilé (Voir G. Mounier, 1942). Les corporations de tanneurs comme celles de Marrakech ou de Fès utilisaient en outre avant l'emploi de produits chimiques, 
de la chaux vive, de la cendre de noyau d'argan, de la crotte de mouton et des cendres de plantes saponaires qui doivent contenir un peu de potasse (voir D. Jemma 1971).

Mais une fois tannée la peau demande un gros travail de martelage, de corroyage et de polissage du côté de la fleur pour les cuirs destinés aux objets décorés et colorés. C'est la qualité des belles basanes du Tafilalet qui ont fait la renommée du cuir dit Filali, apprécié pour la confection des grandes bottes de cavalier, de fourreaux d'armes, de porte-feuilles, de ceintures, de reliures de livre, de tous les objets de maroquinerie.

Sac de voyage touareg pour homme (Photo M. Bovis)

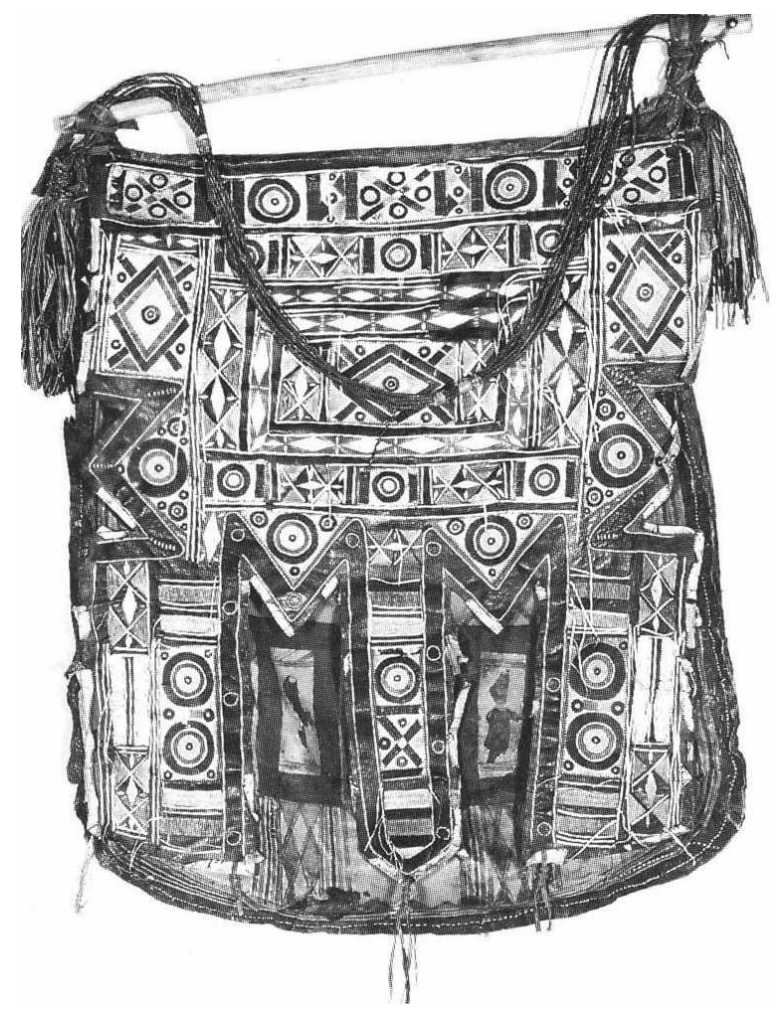

\section{Les colorants}

La peau tannée peut subir un bain de teinture dans la phase finale de sa préparation ou être décorée manuellement et enduite de dessins colorés comme les pratiquent avec un art consommé les femmes mauritaniennes ou touarègues.

Avant l'apparition massive des colorants à l'aniline issus de l'industrie chimique occidentale chaque région utilisait des colorants d'origine locale (minéraux ou végétaux). En pays touareg le blanc était obtenu par un trempage de la peau (fines peaux de chevreau, d'agneau ou de fennec) dans un mélange de graines de mil et d'orge écrasées, de sel et de lait baratté.

La teinte verte était obtenue à partir d'une solution à l'oxyde de cuivre (cuivre rouge, lait baratté, sel d'ammoniaque). Les beaux cuirs verts des sacs et sandales sont tous obtenus ainsi et sont une spécialité des artisans de l'Aïr (šemento). Le rouge était obtenu à partir du pigment rouge de l'Andropogon sorghum avec un mordant de sel ou de natron. Quant à la teinture ocre brun des vélums de tente, elle provenait de carrières d'ocre naturel appelé tameğhoit, alors qu'une teinte semblable est aussi fournie par une 
pierre tendre appelée téfetest. L'ocre jaune est donné par une autre pierre tendre appelée makara. Bien qu'utilisés davantage comme fards pour le visage des femmes ces deux derniers produits peuvent aussi servir pour les peaux. L'écorce de grenade peut donner aussi en macération une teinte jaune clair. Le noir est issu d'un sulfate ferrique cristallisé de couleur gris jaune (tawnkent taneżeżżeft, tafídet ou tafilet en tamâhaq. Voir G. Mounier 1942, p. 140-141). Le bleu indigo était fourni par la plante du même nom cultivée dans les zones sahéliennes (boucle du Niger, Zinder, le Bornou) et importé en Ahaggar sous le nom de bâba (ikoringel).

\section{Usages des peaux et cuirs, symbolisme}

Si l'on dressait l'inventaire des objets d'une tente nomade telle qu'elle existait au début $\mathrm{du}$ siècle, l'on découvrirait que les quatre cinquièmes de cet inventaire sont composés d'objets de cuir. Des outres à contenir les liquides (eau, lait, beurre) à celles contenant les graines, les farines ou semoules, des vaisseaux pour faire boire les animaux, des puisettes pour tirer l'eau des puits, de la tente en cuir, aux tuniques et pantalons de cuir, des lacets, cordes, entraves, petites sacoches et grands sacs de voyage, des chaussures au bouclier, de la selle de chameau à sa rêne, il reste peu d'objets en bois, en métal et en fibre tissée dans le mobilier touareg. Tous les objets possibles et imaginables sont conçus en cuir dans une civilisation qui dispose de peu de bois et n'a pas de métier à tisser.

Bien que la tente touarègue soit le siège de l'âme du groupe (voir H. Hawad-Claudot 1993) à partir duquel s'établissent les rapports avec le monde extérieur et la parenté, la peau elle-même ne semble pas le support de symbolisme particulier dans cette civilisation comme on le remarque au Maroc ou en Kabylie. A Marrakech « la peau de chèvre, en particulier, est mise en gestation dans le ventre maternel figuré par l'iferd, la mare d'eaux en putréfaction, qui représente également le monde souterrain fécondé par les âmes des ancêtres et par Sidi Yaqqub le saint patron... c'est parce que la peau de chèvre a été fécondée dans l'iferd, domaine de l'eau et des morts, qu'elle est chargée de contenir l'eau et d'apporter la pluie» (D. Jemma 1971, p. 59). Dans tout le Maghreb les rogations pour la pluie s'accompagnent de processions montrant la «fiancée de la pluie » qui est une grosse cuillère (ou louche) en bois habillée en femme. Dans bien des cas, elle est vêtue d'une peau de chèvre chargée « d'amener sur terre la pluie et la vie ». En Kabylie, J. Servier signale que lors de l'Aïd el Kebir, le chef de famille enveloppe un moment de la peau sanglante du bélier le dernier de ses enfants « comme pour affirmer son entrée dans la famille » (J. Servier, 1962, p. 262).

Battre le grand tambour chez les Gnawas du Maroc «c'est enfermer dans le cercle de peau, les esprits, qu'il s'agit de maitriser pour les disperser ensuite dans l'enceinte de la maison où se déroulent les danses de possessions " (D. Jemma 1971, p. 60). La peau ici représente le ciel où siègent les esprits qui descendent sur la terre. D'une façon générale, le cuir est symbole de protection ; la chaussure de cuir en est un exemple avec toutes les forces attribuées au pied.

Matière aux multiples usages autrefois, le cuir a perdu de son omniprésence pour devenir un produit de luxe réservé aux plus riches qui redécouvrent avec volupté les vêtements de cuir (blousons, manteaux fourrés, gilets et pantalons) ou aux artistes et poètes qui écrivent le berbère, comme Hawad, sur des peaux de mouton longuement polies au galet. 


\section{BIBLIOGRAPHIE}

BRUNOT L., « Vocabulaire de la tannerie indigène à Rabat », Hespéris, 1923.

BRUNOT L., « La cordonnerie indigène à Rabat », Hespéris, 1946, p. 227-321.

CAMPS G., Massinissa ou les débuts de l'Histoire, Libyca (Archéologie, Epigraphie), t. VIII, 1960, 320 p. pp. 102-111.

CAMPS G., Aux origines de la Berbérie. Monuments et rites funéraires protohistoriques, Paris, A.M.G. 1961, 628 p. pp. $472-73$

GABUS J., Au Sahara, arts et symboles. Ed. de la Baconnière, Neuchatel, 1958, 408 p.

CLAUDOT-HAWAD H., Touaregs, Portrait en fragments, Aix-en-Provence, Edisud, 1993,216p.

GAST M., Alimentation des populations de l'Ahaggar, étude ethnographique, Mémoires du CRAPE VIII

(Alger), Paris, A.M.G., 1968, 458 p.

GASELL S., Hérodote, Alger, A. Jourdan, 1915.

JEMMA D., Les tanneurs de Marrakech, Mémoire du CRAPE XIX, Alger, 1971, 144 p.

LE CORRE F., Le vêtement dans l'art rupestre nord-africain et saharien, Mémoire de DEA, sous la direction du prof. G. Camps, Aix-en-Provence, 1984, 54 p. XXIX pl.

LETOURNEAU R., et Paye L., " La corporation des tanneurs et l'industrie de la tannerie à Fès », Hespéris, t. XXI, 1935, p. 167-239.

LETOURNEAU R., et Paye L., « Les cordonniers de Fès », Hespéris, t. XXIII, 1939, p. 9-54.

MONOD Th., Notes sur le harnachement chamelier, Bulletin de l'IFAN, t. XXIX, série B, 1-2, 1967, pp. 234-274, 58 pl.

MOUNIER G, « Le travail des peaux chez les Touareg du Hoggar », Trav. de l'Institut de recherches Sahariennes, Alger, 1942, t. I, 133-169.

SERVIER J., Les Portes de l'année, Paris, R. Laffont, 1962, 428 p.

SPRUYTTE J., Études expérimentales sur l'attelage, Paris, Crépin-Leblond, 1977, 144 p.

\section{INDEX}

Mots-clés : Artisanat 